\title{
Extracción de almidón de malanga blanca, una alternativa socioambiental para la producción de bases poliméricas biodegradables
}

(c) (i) ()

\section{Malanga blanca starch extraction a socio-environmental alternative for the production of biodegradable polymeric bases}

Juan Carlos González García ${ }^{1}$, Sofía Carolina Godoy Ponce ${ }^{2}$, Alejandro Heredia Jara ${ }^{3}$ \& Lesslie Jokassta Pulgar Astudillo. ${ }^{4}$

Recibido: 10-04-2020/ Revisado: 15-05-2020/Aceptado: 06-06-2020/ Publicado: 03-07-2020

Resumen.

DOI: https://doi.org/10.33262/concienciadigital.v3i3.1263

Colocasia esculenta o malanga blanca es un tubérculo cuyo principal constituyente son los azúcares, se ensayó dos métodos sencillos y económicos de extracción de su almidón utilizando ácido cítrico al 3\% (método 1) y agua destilada (método 2) respectivamente, con el propósito de determinar el procedimiento con mayor rendimiento, el almidón obtenido fue lavado, secado y caracterizado, después, se elaboraron láminas de bases poliméricas combinando el almidón extraído por ambos métodos con diferentes dosis de aditivos con cualidades extensoras, plastificantes, estabilizantes y humectantes, obteniéndose varios prototipos, mismos que fueron sometidos a diferentes ensayos para identificar sus cualidades y degradabilidad, los análisis de varianza aplicados determinaron que el rendimiento en la extracción del almidón dependen del método utilizado, obteniéndose valores más altos cercanos al 28\% para el método 1; dentro de la caracterización del almidón resalta que solamente el contenido de humedad tenía variación en dependencia del método usado y el resto de propiedades medidas no guardaban relación, es decir no dependían del método de extracción del almidón; las láminas poliméricas con combinaciones A3B3C1 (glicerina $6 \mathrm{~mL}+0,75 \mathrm{~g}$ carboximetilcelulosa $+2,5 \mathrm{~mL}$ ácido acético) elaboradas con

\footnotetext{
${ }^{1}$ Escuela Superior Politécnica de Chimborazo, Riobamba, Ecuador, juan.gonzalez@espoch.edu.ec

${ }^{2}$ Escuela Superior Politécnica de Chimborazo, Riobamba, Ecuador, sofia.godoy@espoch.edu.ec

${ }^{3}$ Investigador autónomo, Riobamba, Ecuador, dannyahj@hotmail.com

${ }^{4}$ Investigador autónomo, Riobamba, Ecuador, lessliejokassta@gmail.com
} 
almidón extraído por el método 1 y A1B3C2 (glicerina 2,5mL+0,75 g carboximetilcelulosa+4mL ácido acético) elaboradas con almidón extraído por el método 2 mostraron mejores cualidades físicas de homogeneidad en su textura, consistencia y ausencia de grumos; respecto a los ensayos de degradabilidad se verificó que en un promedio de 130 días en el suelo y 33 días en el agua fueron suficientes para degradar totalmente las láminas; desde el punto de vista social producir almidón de malanga por el método 1 beneficiaría a las comunidades productoras dando un valor agregado al mismo y ambientalmente dadas las características de las bases poliméricas obtenidas podría sustituirse parcial o totalmente en la elaboración de ciertos productos que en la actualidad utilizan polímeros sintéticos.

Palabras claves: colocasia esculenta, malanga blanca, bases poliméricas biodegradables, extracción de almidón, desarrollo socioambiental.

\begin{abstract}
.
Colocasia esculenta or white Malanga is a tuber whose main constituent is sugars, two simple and inexpensive methods of extracting its starch were tried using $3 \%$ citric acid (method 1) and distilled water (method 2) respectively, in order to determine the procedure with the highest yield, the starch obtained was washed, dried and characterized, then, sheets of polymeric bases were made combining the starch extracted by both methods with different doses of additives with extensor, plasticizing, stabilizing and humectant qualities, obtaining various prototypes., which were subjected to different tests to identify their qualities and degradability, the applied variance analyzes determined that the starch extraction performance depends on the method used, obtaining higher values close to $28 \%$ for method 1; within the characterization of the starch, it stands out that only the moisture content had variation depending on the method used and the rest of the measured properties were not related, that is, they did not depend on the starch extraction method; polymeric sheets with combinations A3B3C1 (glycerin $6 \mathrm{~mL}+0.75 \mathrm{~g}$ carboxymethylcellulose $+2.5 \mathrm{~mL}$ acetic acid) made with starch extracted by method 1 and A1B3C2 (glycerin $2.5 \mathrm{~mL}+0.75 \mathrm{~g}$ carboxymethylcellulose $+4 \mathrm{~mL}$ acetic acid) made with starch extracted by method 2 showed better physical qualities of homogeneity in its texture, consistency and absence of lumps; Regarding the degradability tests, it was verified that an average of 130 days in the soil and 33 days in the water were sufficient to completely degrade the sheets; from a social point of view, producing taro starch by method 1 would benefit the producing communities, giving added value to it, and environmentally given the characteristics of the polymer bases obtained, it could be partially or totally substituted in the elaboration of certain products that currently they use synthetic polymers.
\end{abstract}


Keywords: colocasia esculenta, white malanga, biodegradable polymeric bases, starch extraction, socio-environmental development.

\section{Introducción}

Dentro de los cultivos no tradicionales en el Ecuador destaca la malanga blanca, que en los últimos años ha aumentado su producción y es destinada exclusivamente a la exportación sin recibir ningún proceso que le añada valor, estudios realizados han determinado que éste tubérculo contiene altos porcentajes de almidón, componente de alta demanda debido a sus múltiples aplicaciones y usos en la industria alimenticia, de fármacos, cosmética e incluso en la producción de polímeros naturales, la malanga blanca requiere para su producción de bajas inversiones con altas rentabilidades. (Fuentes-Zaragoza, 2010).

En el país, el rendimiento en la producción a gran escala de malanga es de alrededor de 15 ton/Ha obteniéndose diferentes calidades de producto, solo las de óptima calidad (10 ton) pueden ser exportadas, desechándose el resto (5 ton) ya que no consiguen ser comercializadas, por tal razón la necesidad de su aprovechamiento.

La producción de malanga blanca en parcelas comunitarias tiene bajos costos, oscilan alrededor de 150-200 dólares por hectárea con rendimientos productivos de 2-2,5 toneladas por hectárea. (MAGAP, 2012)

Utilizando tecnologías sencillas, es posible aprovechar este recurso agrícola extrayendo su almidón, el método de Brenda que utiliza una solución de ácido cítrico para la extracción alcanza rendimientos aceptables con bajas inversiones. (Heredia, Pulgar, 2019)

El valor de una caja exportable de 40 libras de malanga blanca en el mercado cuesta alrededor de 10 dólares y en el puerto llega a los 14 dólares, razón por la cual se analiza la posibilidad de darle un valor agregado al producto para mejorar los ingresos de las comunidades productoras tomando en cuenta que extraer $1 \mathrm{~kg}$ de almidón de malanga por métodos sencillos significa una inversión de 2 dólares y su valor en el mercado puede alcanzar fácilmente 5-8 dólares/ kg. (Heredia, Pulgar, 2019)

Las comunidades rurales de la región amazónica ecuatoriana tienen alta incidencia de pobreza y son justamente aquellos lugares donde se cultiva y produce malanga blanca en la actualidad, de acuerdo a datos del INEC (2010) tiene una extrema pobreza y una pobreza por necesidades básicas insatisfechas de la población del 57\% y el 99\% respectivamente.

\section{Metodología}

Mediante muestreo no probabilístico discrecional se obtuvieron frutos (raíces) de malanga blanca producidas en la región amazónica ecuatoriana, posteriormente se prepararon las muestras para la extracción de su almidón. 
Figura 1: Selección de los frutos de malanga blanca

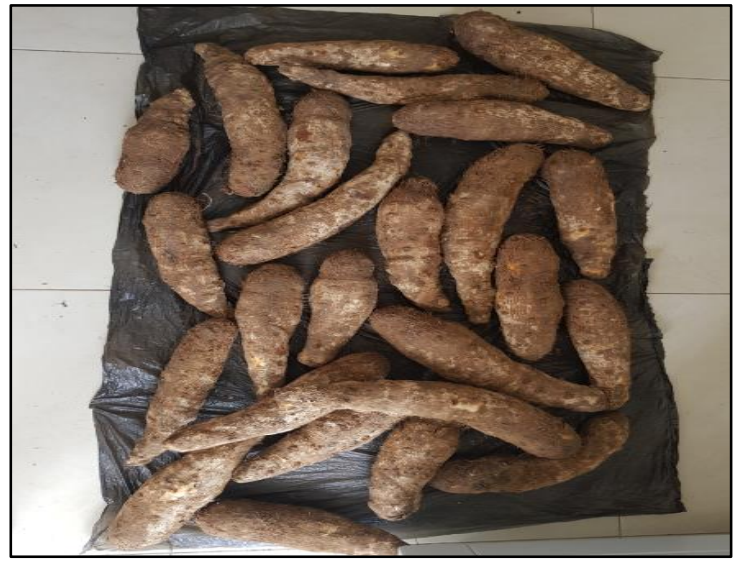

Fuente: Heredia, D. Pulgar, L. 2019.

\section{Extracción de almidón}

Se ensayaron dos metodologías de extracción del almidón de malanga, el primero con ácido cítrico al 3\% (método 1) y el segundo con agua destilada (método 2).

\section{Para el método 1:}

- Se pelaron y cortaron en cubos de alrededor de $5 \mathrm{~cm}$ de lado.

- Se introdujeron en una solución de ácido cítrico al 3\%.

- Seguidamente se licuó la pulpa hasta desintegración total.

- Se tamizó y enjuagó el producto en mallas de $200 \mu \mathrm{m}$ hasta obtención de que el agua clara.

- Se sedimentó durante 24 horas.

- La torta retenida se maceró, tamizó y sedimentó nuevamente.

- El almidón obtenido se secó, pulverizó y finalmente almacenó. (Chávez, 2015, p. 39).

\section{Para el método 2:}

- Se lavó, peló y cortó las raíces.

- Se licuó con agua destilada por 2 minutos.

- Se dejó en reposo una hora para obtener el material en suspensión.

- Se filtró y lavó repetidamente.

- Se dejó reposar por 2 horas la solución.

- Se tamizó y secó a $40^{\circ} \mathrm{C}$. por 24 horas.

- Se trituró, tamizó y almacenó herméticamente el almidón obtenido. (Fallis, 2013, p. 16891699). 


\section{Análisis proximal del almidón de malanga}

Para el análisis proximal de caracterización se trabajó por separado para al almidón extraído por los dos métodos.

Tabla 1: Análisis proximal del almidón de malanga blanca.

\begin{tabular}{|c|c|c|}
\hline Norma & Técnica & Equipo \\
\hline $\begin{array}{l}\text { (NTE INEN } \\
1513:, 1987) .\end{array}$ & $\begin{array}{c}\text { Determinación de \% de materia seca y } \\
\text { humedad. }\end{array}$ & Estufa de aire caliente. \\
\hline $\begin{array}{c}- \\
\text { (INEN, 2013). } \\
\text { (NTE INEN } \\
\text { 0519, 1981) }\end{array}$ & $\begin{array}{l}\text { Determinación de humedad (Bianco, } \\
\text { Capote y Garbendia, 2014, p. 29-36). } \\
\text { Determinación de ceniza. } \\
\text { Determinación de proteína cruda }\end{array}$ & $\begin{array}{l}\text { Termobalanza } \\
\text { Mufla } \\
\text { Macrokjeldhal }\end{array}$ \\
\hline $\begin{array}{c}- \\
\text { INEN } 5231980- \\
12 \\
(517,1980, \text { p. } 1- \\
5) .\end{array}$ & $\begin{array}{c}\text { Determinación de (E) extracto libre no } \\
\text { nitrogenado (Guevara, 2016, p. 73). } \\
\text { Determinación de extracto etéreo } \\
\text { (E.E.) }\end{array}$ & $\begin{array}{c}\% \mathrm{E}=100-\Sigma[\% \mathrm{H}+\% \mathrm{C}+\% \mathrm{~F}+\% \mathrm{E} . \mathrm{E} \\
+\% \mathrm{P}] \\
\text { Soxhlet. }\end{array}$ \\
\hline $\begin{array}{l}\text { (INEN NTE } \\
0522,1981)\end{array}$ & Determinación de fibra cruda $(\mathrm{F})$ & Weende. \\
\hline- & $\begin{array}{l}\text { Determinación de almidón (Química, } \\
\qquad 2008 \text { ). }\end{array}$ & Espectrofotómetro. \\
\hline- & $\begin{array}{l}\text { Determinación de amilosa (Salvador, } \\
\qquad 2013 \text { ). }\end{array}$ & Espectrofotómetro. \\
\hline- & $\begin{array}{l}\text { Determinación amilopectina (Gonz, } \\
\qquad 2018 \text {, p. } 35-44) \text {. }\end{array}$ & $\begin{array}{l}\text { Por diferencia entre la cantidad de } \\
\text { almidón y amilosa. }\end{array}$ \\
\hline
\end{tabular}

Fuente: Heredia, D. Pulgar, L. 2019.

\section{Dosificación de aditivos.}

En la siguiente tabla se presentan las dosificaciones de aditivos que se utilizaron en la elaboración de láminas de bases poliméricas:

Tabla 2: Dosificación de aditivos para obtención de láminas de bases poliméricas.

\begin{tabular}{cccccc}
\hline \multicolumn{3}{l}{ Componentes constantes } & \multicolumn{3}{c}{ Componentes variables } \\
\hline $\mathbf{H}_{2} \mathrm{O}$ & $\mathrm{NaCl}$ & Aceite de & Aditivo A & Aditivo B & Aditivo C \\
$(\%)$ & $(\%)$ & Girasol & $(\%)$ & $(\%)$ & $(\%)$ \\
& & $(\%)$ & & & 2.5 \\
\hline
\end{tabular}




\begin{tabular}{lllccc}
\hline 40 & 1 & 0.5 & 2.5 & 0.25 & 2.5 \\
40 & 1 & 0.5 & 2.5 & 0.25 & 2.5 \\
40 & 1 & 0.5 & 4 & 0.5 & 4 \\
40 & 1 & 0.5 & 4 & 0.5 & 4 \\
40 & 1 & 0.5 & 4 & 0.5 & 4 \\
40 & 1 & 0.5 & 6 & 0,75 & 6 \\
40 & 1 & 0.5 & 6 & 0,75 & 6 \\
40 & 1 & 0.5 & 6 & 0,75 & 6
\end{tabular}

Aditivo A: glicerina; Aditivo B: CMC carboximetilcelulosa; Aditivo C: ácido acético.

Fuente: Heredia, D. Pulgar, L. 2019.

Se elaboró un diseño de bloques completamente al azar con tres factores.

Tabla 3: Combinaciones experimentales con el almidón extraído por el método 1.

\begin{tabular}{|c|c|c|}
\hline A1 & A2 & $\mathbf{A 3}$ \\
\hline A1B1C1 & $\mathrm{A} 2 \mathrm{~B} 1 \mathrm{C} 1$ & A3B1C1 \\
\hline $\mathrm{A} 1 \mathrm{~B} 1 \mathrm{C} 2$ & $\mathrm{~A} 2 \mathrm{~B} 1 \mathrm{C} 2$ & $\mathrm{~A} 3 \mathrm{~B} 1 \mathrm{C} 2$ \\
\hline $\mathrm{A} 1 \mathrm{~B} 1 \mathrm{C} 3$ & $\mathrm{~A} 2 \mathrm{~B} 1 \mathrm{C} 3$ & $\mathrm{~A} 3 \mathrm{~B} 1 \mathrm{C} 3$ \\
\hline $\mathrm{A} 1 \mathrm{~B} 2 \mathrm{C} 1$ & $\mathrm{~A} 2 \mathrm{~B} 2 \mathrm{C} 1$ & $\mathrm{~A} 3 \mathrm{~B} 2 \mathrm{C} 1$ \\
\hline $\mathrm{A} 1 \mathrm{~B} 2 \mathrm{C} 2$ & $\mathrm{~A} 2 \mathrm{~B} 2 \mathrm{C} 2$ & $\mathrm{~A} 3 \mathrm{~B} 2 \mathrm{C} 2$ \\
\hline A1B2C3 & $\mathrm{A} 2 \mathrm{~B} 2 \mathrm{C} 3$ & A3B2C 3 \\
\hline A1B3C1 & $\mathrm{A} 2 \mathrm{~B} 3 \mathrm{C} 1$ & A3B3C1 \\
\hline $\mathrm{A} 1 \mathrm{~B} 3 \mathrm{C} 2$ & $\mathrm{~A} 2 \mathrm{~B} 3 \mathrm{C} 2$ & A3B3C2 \\
\hline A1B3C3 & $\mathrm{A} 2 \mathrm{~B} 3 \mathrm{C} 3$ & A3B3C 3 \\
\hline \multicolumn{3}{|c|}{$\begin{array}{l}\text { A1 (2,5\% glicerina), A2 ( } 4 \% \text { glicerina), A3 ( } 6 \% \text { glicerina), B1 ( } 0,25 \% \text { CMC), } \\
\text { B2 ( } 0,5 \% \text { CMC), B3 ( } 0,75 \% \text { CMC), C1 ( } 2,5 \% \text { ácido acético), C2 ( } 4 \% \text { ácido } \\
\text { acético), C3 ( } 6 \% \text { ácido acético). }\end{array}$} \\
\hline
\end{tabular}

Fuente: (González, 2018, p. 35-44).

Determinación de las propiedades de las láminas poliméricas.

Tabla 4: Técnicas de determinación de las propiedades de las bases poliméricas.

\begin{tabular}{clc}
\hline Propiedad & \multicolumn{1}{c}{ Técnica } & \multicolumn{1}{c}{ Equipos } \\
\hline \multirow{5}{*}{ Química } & Degradación en suelo (Râpă et al., 2014, p. 34-42). & Cajas petri \\
& $\begin{array}{l}\text { Degradación hidrolítica (Karina, 2013). } \\
\text { Degradación térmica (González, 2018, p. 35-44). } \\
\text { Solubilidad en agua (Autor y Alejandro Moreno Toasa, }\end{array}$ & Estufa de aire caliente \\
& $\begin{array}{l}\text { 2015). } \\
\text { Funcional }\end{array}$ & $\begin{array}{c}\text { Balanza analítica } \\
\text { Estufa }\end{array}$ \\
& $\begin{array}{l}\text { Absorción de agua (González, 2018, p. 35-44). } \\
\text { Temperatura de gelatinización (González, 2018, p. 35- } \\
\text { 44). }\end{array}$ & Balanza analítica \\
& & Estufa de aire caliente \\
\hline
\end{tabular}

Fuente: Heredia, D.; Pulgar, L. 2019. 


\section{Resultados}

\section{Rendimiento del almidón.}

En las tablas siguientes se exponen los resultados obtenidos del rendimiento de almidón obtenidos por ambos métodos.

Tabla 5: Rendimiento de extracción por el método 1.

\begin{tabular}{ccccc}
\hline Medición & $\begin{array}{c}\text { Malanga } \\
\text { con cáscara } \\
(\mathbf{g})\end{array}$ & $\begin{array}{c}\text { Malanga sin } \\
\text { cáscara } \\
(\mathbf{g})\end{array}$ & $\begin{array}{c}\text { Almidón } \\
(\mathbf{g})\end{array}$ & $\begin{array}{c}\text { Rendimiento } \\
(\mathbf{\%})\end{array}$ \\
\hline A1 & & & 740,69 & 29,62 \\
A2 & $25.000,00$ & $6.859,68$ & 715,08 & 28,60 \\
A3 & & & 561,81 & 22,47 \\
B1 & & & 762,30 & 30,49 \\
B2 & $25.000,00$ & $6.859,68$ & 722,10 & 28,88 \\
B3 & & & 568,70 & 22,74 \\
C1 & & & 756,50 & 30,26 \\
C2 & $25.000,00$ & $6.859,68$ & 718,89 & 28,75 \\
C3 & & & 559,70 & 22,38 \\
Promedio & $\mathbf{2 5 . 0 0 0 , 0 0}$ & $\mathbf{6 . 8 5 9 , 6 8}$ & $\mathbf{6 7 8 , 4 1}$ & $\mathbf{2 7 , 1 3}$ \\
\hline
\end{tabular}

Fuente: Heredia, D.; Pulgar, L. 2019.

Tabla 6: Rendimiento de extracción por el método 2.

\begin{tabular}{ccccc}
\hline Medición & $\begin{array}{c}\text { Malanga } \\
\text { con cáscara } \\
(\mathbf{g})\end{array}$ & $\begin{array}{c}\text { Malanga sin } \\
\text { cáscara } \\
(\mathbf{g})\end{array}$ & $\begin{array}{c}\text { Almidón } \\
(\mathbf{g})\end{array}$ & $\begin{array}{c}\text { Rendimiento } \\
(\mathbf{\%})\end{array}$ \\
\hline D1 & & & 331,94 & 13,27 \\
D2 & $25.000,00$ & $6.859,68$ & 344,00 & 13,76 \\
D3 & & & 338,13 & 13,52 \\
E1 & & & 337,60 & 13,50 \\
E2 & $25.000,00$ & $6.859,68$ & 339,34 & 13,57 \\
E3 & & & 335,23 & 13,40 \\
F1 & & & 333,46 & 13,33 \\
F2 & $25.000,00$ & $6.859,68$ & 346,67 & 13,86 \\
F3 & & & 336,21 & 13,44 \\
Promedio & $\mathbf{2 5 . 0 0 0 , 0 0}$ & $\mathbf{6 . 8 5 9 , 6 8}$ & $\mathbf{3 3 8 , 0 6}$ & $\mathbf{1 3 , 5 1}$ \\
\hline
\end{tabular}

Fuente: Heredia, D.; Pulgar, L. 2019. 


\section{Caracterización del almidón}

Tabla 7: Análisis proximal del almidón obtenido por el método 1.

\begin{tabular}{cccccccc}
\hline $\begin{array}{c}\text { Medici } \\
\text { ón }\end{array}$ & $\begin{array}{c}* \text { Humeda } \\
\mathbf{d} \\
(\boldsymbol{\%})\end{array}$ & $\begin{array}{c}* * \text { Humeda } \\
\mathbf{d}\end{array}$ & $\begin{array}{c}\text { Cenizas } \\
\mathbf{\%})\end{array}$ & $\begin{array}{c}\text { Proteín } \\
\mathbf{9}\end{array}$ & $\begin{array}{c}\text { Grasa } \\
\mathbf{\%}\end{array}$ & $\begin{array}{c}\text { Fibra } \\
\text { cruda } \\
\mathbf{\%}\end{array}$ & $\begin{array}{c}\text { E.N } \\
(\mathbf{\%})\end{array}$ \\
\hline A1 & 14,06 & 10,99 & 0,73 & 2,36 & 0,039 & 0,29 & 82,521 \\
A2 & 14,02 & 11,13 & 0,59 & 2,17 & 0,049 & 0,35 & 82,821 \\
A3 & 13,94 & 11,32 & 0,61 & 2,18 & 0,044 & 0,31 & 82,916 \\
B1 & 13,70 & 10,43 & 0,92 & 2,15 & 0,039 & 0,34 & 82,851 \\
B2 & 13,40 & 10,01 & 0,47 & 2,19 & 0,044 & 0,35 & 83,546 \\
B3 & 13,34 & 11,79 & 0,82 & 2,39 & 0,039 & 0,37 & 83,041 \\
C1 & 13,37 & 10,59 & 0,47 & 2,19 & 0,044 & 0,28 & 83,646 \\
C2 & 13,16 & 10,77 & 0,46 & 2,18 & 0,039 & 0,29 & 83,871 \\
C3 & 13,09 & 11,25 & 0,40 & 2,39 & 0,039 & 0,32 & 83,761 \\
media & $\mathbf{1 3 , 5 6}$ & $\mathbf{1 0 , 9 2}$ & $\mathbf{0 , 6 1}$ & $\mathbf{2 , 2 4}$ & $\mathbf{0 , 0 4 2}$ & $\mathbf{0 , 3 2}$ & $\mathbf{8 3 , 2 1 9}$ \\
& & & & & & \\
*Termobalanza, **Estufa de aire caliente & & & & & \\
\hline
\end{tabular}

Fuente: Heredia, D.; Pulgar, L. 2019.

Tabla 8: Análisis proximal del almidón obtenido por el método 2.

\begin{tabular}{|c|c|c|c|c|c|c|c|}
\hline $\begin{array}{l}\text { Medici } \\
\text { ón }\end{array}$ & $\begin{array}{c}\text { Humedad } \\
(\%)\end{array}$ & $\begin{array}{c}\text { Humedad } \\
(\%)\end{array}$ & $\begin{array}{c}\text { Ceniza } \\
\%\end{array}$ & $\begin{array}{c}\text { Proteí } \\
\text { na } \\
\%\end{array}$ & $\begin{array}{c}\text { Grasa } \\
\%\end{array}$ & $\begin{array}{c}\text { Fibra } \\
\text { cruda } \\
\%\end{array}$ & E.N (\%) \\
\hline D1 & 19,41 & 15,31 & 0,49 & 2,63 & 0,044 & 0,29 & 77,136 \\
\hline D2 & 19,17 & 15,49 & 0,47 & 2,42 & 0,049 & 0,31 & 77,611 \\
\hline D3 & 18,79 & 15,99 & 0,94 & 2,31 & 0,049 & 0,33 & 77,581 \\
\hline E1 & 20,26 & 14,87 & 0,95 & 2,61 & 0,039 & 0,36 & 75,781 \\
\hline E2 & 19,88 & 14,84 & 0,74 & 2,62 & 0,044 & 0,35 & 76,366 \\
\hline E3 & 19,31 & 14,55 & 0,65 & 2,41 & 0,049 & 0,28 & 77,301 \\
\hline $\mathrm{F} 1$ & 19,71 & 15,70 & 0,34 & 2,41 & 0,039 & 0,33 & 77,171 \\
\hline F2 & 19,59 & 16,02 & 0,35 & 2,58 & 0,044 & 0,31 & 77,126 \\
\hline F3 & 19,61 & 14,62 & 0,39 & 2,60 & 0,044 & 0,32 & 77,036 \\
\hline media & 19,53 & 15,27 & 0,59 & 2,51 & 0,045 & $\mathbf{0 , 3 2}$ & 77,012 \\
\hline
\end{tabular}

Fuente: Heredia, D.; Pulgar, L. 2019. 
Tabla 9: Porcentaje de amilosa y amilopectina en almidón extraído por los dos métodos.

\begin{tabular}{ccc}
\hline \multicolumn{2}{c}{ Método 1 } \\
\hline Medición & \% Amilosa & \% Amilopectina \\
\hline A1 & 27,28 & 72,72 \\
A2 & 26,24 & 73,76 \\
A3 & 26,76 & 73,24 \\
B1 & 26,30 & 73,70 \\
B2 & 25,26 & 74,74 \\
B3 & 25,78 & 74,22 \\
C1 & 28,26 & 71,74 \\
C2 & 27,22 & 72,78 \\
C3 & 27,74 & 72,26 \\
Promedio & $\mathbf{2 6 , 7 6}$ & $\mathbf{7 3 , 2 4}$ \\
& & Método 2 \\
D1 & 25,30 & 74,70 \\
D2 & 24,26 & 75,74 \\
D3 & 24,78 & 75,22 \\
E1 & 25,99 & 74,01 \\
E2 & 24,95 & 75,05 \\
E3 & 25,47 & 74,53 \\
F1 & 24,62 & 75,38 \\
F2 & 23,58 & 76,42 \\
F3 & 24,10 & 75,90 \\
Promedio & $\mathbf{2 4 , 7 8}$ & $\mathbf{7 5 , 2 1}$ \\
\hline
\end{tabular}

Fuente: Heredia, D.; Pulgar, L. 2019.

Tabla 10: ANAVA, composición del almidón extraído por el método 1 y 2.

\begin{tabular}{|c|c|c|c|c|c|}
\hline Método & Análisis & $\begin{array}{c}\text { Fisher } \\
\text { calculado } \\
\text { (F) }\end{array}$ & p-valor & Medias & Significancia \\
\hline 1 & $\begin{array}{c}\% \mathrm{R} \\
\text { Rendimiento almidón }\end{array}$ & 34,54 & 0,0042 & $\begin{array}{l} \pm 27,37 \\
\pm 13,49\end{array}$ & $* *$ \\
\hline 1 & $\% \mathrm{H}$ & & & $\pm 14,01$ & \\
\hline 2 & $\begin{array}{c}\text { Humedad } \\
\text { termobalanza }\end{array}$ & 774,06 & $<0,0001$ & $\pm 19,12$ & $* *$ \\
\hline 2 & $\begin{array}{c}\% \mathrm{H} \\
\text { Humedad estufa }\end{array}$ & 53,78 & $<0,0018$ & $\begin{array}{l} \pm 10,74 \\
\pm 14,75\end{array}$ & $* *$ \\
\hline $\begin{array}{l}1 \\
2\end{array}$ & $\begin{array}{c}\% \mathrm{C} \\
\text { cenizas }\end{array}$ & 0,07 & 0,8033 & $\begin{array}{l} \pm 0,74 \\
\pm 0.78\end{array}$ & ns \\
\hline
\end{tabular}




\begin{tabular}{|c|c|c|c|c|c|}
\hline 1 & $\% \mathrm{P}$ & \multirow{2}{*}{3,72} & \multirow{2}{*}{0,1260} & $\pm 2,24$ & \multirow[b]{2}{*}{ ns } \\
\hline 2 & proteína & & & $\pm 2,45$ & \\
\hline 1 & $\% \mathrm{G}$ & \multirow{2}{*}{1,00} & \multirow{2}{*}{0,3739} & $\pm 0,04$ & \multirow{3}{*}{ ns } \\
\hline 2 & grasa & & & $\pm 0,04$ & \\
\hline 1 & $\% \mathrm{~F}$ & \multirow{3}{*}{3,06} & \multirow{3}{*}{0,1550} & $\pm 0,30$ & \\
\hline 2 & fibra & & & $\pm 0,32$ & \multirow[t]{2}{*}{ ns } \\
\hline 1 & (E.N) & & & $\pm 83,76$ & \\
\hline 2 & $\begin{array}{c}\text { Extracto libre no } \\
\text { nitrogenado }\end{array}$ & 7628,05 & $<0,0001$ & $\pm 77,11$ & $* *$ \\
\hline 1 & \multirow{2}{*}{$\%$ amilosa } & \multirow{2}{*}{0,53} & \multirow{2}{*}{0,5058} & $\pm 25,78$ & \multirow{3}{*}{ ns } \\
\hline 2 & & & & $\pm 25,47$ & \\
\hline 1 & \multirow[b]{2}{*}{$\%$ amilopectina } & \multirow{2}{*}{0,53} & \multirow{2}{*}{0,5058} & $\pm 74,53$ & \\
\hline 2 & & & & $\pm 74,22$ & ns \\
\hline 1 & \multirow{2}{*}{ \% almidón } & \multirow{2}{*}{19,94} & \multirow{2}{*}{0,0111} & $\pm 74,06$ & \\
\hline 2 & & & & $\pm 65,21$ & * \\
\hline
\end{tabular}

Fuente: Heredia, D.; Pulgar, L. 2019.

El rendimiento en la extracción de almidón depende del método usado, ya que el p-valor es altamente significativo, el método 1 es más factible porque se obtuvo un rendimiento cercano al $28 \%$ vs un $14 \%$ obtenido para el almidón extraído por el método 2.

Puede apreciarse que, dentro de los parámetros determinados en el análisis proximal del almidón de malanga extraído, algunos tienen dependencia del método y otros no, por tanto, dependiendo del parámetro de interés se podría decidir el método de extracción a usar.

\section{Dosificación de aditivos}

Se elaboraron laminas poliméricas combinando almidón + aditivos y se obtuvieron las características que se muestran en la tabla siguiente:

Tabla 11: Características de láminas poliméricas.

\begin{tabular}{|c|c|c|c|}
\hline \multirow{2}{*}{ \# } & \multirow{2}{*}{ Combinación } & \multicolumn{2}{|c|}{ Características } \\
\hline & & Método 1 & Método 2 \\
\hline 1 & $\mathrm{~A} 1 \mathrm{~B} 1 \mathrm{C} 1$ & $\begin{array}{l}\text { Densidad alta, homogeneidad media, } \\
\text { grumosidad baja. }\end{array}$ & $\begin{array}{l}\text { Densidad alta, homogeneidad } \\
\text { media, grumosidad baja. }\end{array}$ \\
\hline 2 & $\mathrm{~A} 1 \mathrm{~B} 1 \mathrm{C} 2$ & $\begin{array}{l}\text { Densidad media, homogeneidad } \\
\text { media, grumosidad baja. }\end{array}$ & $\begin{array}{l}\text { Densidad media, homogeneidad } \\
\text { media, grumosidad baja. }\end{array}$ \\
\hline 3 & A1B1C3 & $\begin{array}{c}\text { Densidad baja, homogeneidad alta, } \\
\text { grumosidad baja. }\end{array}$ & $\begin{array}{l}\text { Densidad baja, homogeneidad } \\
\text { alta, grumosidad baja. }\end{array}$ \\
\hline 4 & $\mathrm{~A} 1 \mathrm{~B} 2 \mathrm{C} 1$ & $\begin{array}{l}\text { Densidad alta, homogeneidad baja, } \\
\text { grumosidad media }\end{array}$ & $\begin{array}{l}\text { Densidad alta y homogeneidad } \\
\text { media, grumosidad alta. }\end{array}$ \\
\hline
\end{tabular}




\begin{tabular}{|c|c|c|c|}
\hline 5 & $\mathrm{~A} 1 \mathrm{~B} 2 \mathrm{C} 2$ & $\begin{array}{l}\text { Densidad media, homogeneidad } \\
\text { media, grumosidad media. }\end{array}$ & $\begin{array}{l}\text { Densidad media, homogeneidad } \\
\text { alta, grumosidad baja. }\end{array}$ \\
\hline 6 & A1B2C3 & $\begin{array}{c}\text { Densidad baja, homogeneidad alta, } \\
\text { grumosidad baja. }\end{array}$ & $\begin{array}{l}\text { Densidad baja, homogeneidad } \\
\text { alta, grumosidad baja. }\end{array}$ \\
\hline 7 & A1B3C1 & $\begin{array}{l}\text { Densidad alta, homogeneidad baja, } \\
\text { grumosidad alta. }\end{array}$ & $\begin{array}{l}\text { Densidad alta, homogeneidad } \\
\text { baja, grumosidad baja. }\end{array}$ \\
\hline 8 & $\mathrm{~A} 1 \mathrm{~B} 3 \mathrm{C} 2$ & $\begin{array}{l}\text { Densidad alta, homogeneidad media, } \\
\text { grumosidad media. }\end{array}$ & $\begin{array}{l}\text { Densidad media, homogeneidad } \\
\text { alta, grumosidad baja. }\end{array}$ \\
\hline 9 & A1B3C3 & $\begin{array}{l}\text { Densidad alta, homogeneidad baja, } \\
\text { grumosidad baja. }\end{array}$ & $\begin{array}{l}\text { Densidad media, homogeneidad } \\
\text { media, grumosidad baja. }\end{array}$ \\
\hline 10 & $\mathrm{~A} 2 \mathrm{~B} 1 \mathrm{C} 1$ & $\begin{array}{l}\text { Densidad media, homogeneidad alta, } \\
\text { grumosidad baja. }\end{array}$ & $\begin{array}{l}\text { Densidad media, homogeneidad } \\
\text { alta, grumosidad baja. }\end{array}$ \\
\hline 11 & $\mathrm{~A} 2 \mathrm{~B} 1 \mathrm{C} 2$ & $\begin{array}{c}\text { Densidad baja, homogeneidad alta, } \\
\text { grumosidad baja. }\end{array}$ & $\begin{array}{l}\text { Densidad baja, homogeneidad } \\
\text { alta, grumosidad baja. }\end{array}$ \\
\hline 12 & A2B1C3 & $\begin{array}{l}\text { Densidad baja, homogeneidad alta, } \\
\text { grumosidad baja. }\end{array}$ & $\begin{array}{l}\text { Densidad baja, homogeneidad } \\
\text { alta, grumosidad baja. }\end{array}$ \\
\hline 13 & $\mathrm{~A} 2 \mathrm{~B} 2 \mathrm{C} 1$ & $\begin{array}{l}\text { Densidad baja, homogeneidad media, } \\
\text { grumosidad baja. }\end{array}$ & $\begin{array}{l}\text { Densidad alta, homogeneidad } \\
\text { media, grumosidad baja. }\end{array}$ \\
\hline 14 & $\mathrm{~A} 2 \mathrm{~B} 2 \mathrm{C} 2$ & $\begin{array}{l}\text { Densidad baja, homogeneidad media, } \\
\text { grumosidad baja. }\end{array}$ & $\begin{array}{l}\text { Densidad baja, homogeneidad } \\
\text { alta, grumosidad baja. }\end{array}$ \\
\hline 15 & $\mathrm{~A} 2 \mathrm{~B} 2 \mathrm{C} 3$ & $\begin{array}{l}\text { Densidad baja, homogeneidad media, } \\
\text { grumosidad baja. }\end{array}$ & $\begin{array}{l}\text { Densidad baja, homogeneidad } \\
\text { media, grumosidad baja. }\end{array}$ \\
\hline \multicolumn{4}{|c|}{$\begin{array}{l}\text { A1:2,5 mL de glicerina; A2: 4mL glicerina; A3: 6mL glicerina; B1:0,25 CMC; B2:0,50 CMC; } \\
\text { B1:0,75 CMC; C1:2,5 ácido acético; C1:4,0 ácido acético; C1:6,0 ácido acético. }\end{array}$} \\
\hline
\end{tabular}

Fuente: Heredia, D.; Pulgar, L. 2019.

Tabla 12: Características de láminas poliméricas (continuación)

\begin{tabular}{|c|c|c|c|}
\hline \multirow{2}{*}{ \# } & \multirow{2}{*}{ Combinación } & \multicolumn{2}{|c|}{ Características } \\
\hline & & Método 1 & Método 2 \\
\hline 16 & $\mathrm{~A} 2 \mathrm{~B} 3 \mathrm{C} 1$ & $\begin{array}{l}\text { Densidad alta, homogeneidad } \\
\text { media, grumosidad baja. }\end{array}$ & $\begin{array}{l}\text { Densidad alta, homogeneidad } \\
\text { media, grumosidad media. }\end{array}$ \\
\hline 17 & $\mathrm{~A} 2 \mathrm{~B} 3 \mathrm{C} 2$ & $\begin{array}{l}\text { Densidad media, homogeneidad } \\
\text { media, grumosidad baja. }\end{array}$ & $\begin{array}{l}\text { Densidad alta, homogeneidad } \\
\text { baja, grumosidad baja. }\end{array}$ \\
\hline 18 & $\mathrm{~A} 2 \mathrm{~B} 3 \mathrm{C} 3$ & $\begin{array}{c}\text { Densidad baja, homogeneidad alta, } \\
\text { grumosidad alta. }\end{array}$ & $\begin{array}{c}\text { Densidad media, homogeneidad } \\
\text { alta, grumosidad alta. }\end{array}$ \\
\hline 19 & $\mathrm{~A} 3 \mathrm{~B} 1 \mathrm{C} 1$ & $\begin{array}{l}\text { Densidad alta, homogeneidad } \\
\text { media, grumosidad baja. }\end{array}$ & $\begin{array}{c}\text { Densidad media, homogeneidad } \\
\text { alta, grumosidad baja. }\end{array}$ \\
\hline 20 & $\mathrm{~A} 3 \mathrm{~B} 1 \mathrm{C} 2$ & $\begin{array}{l}\text { Densidad media, homogeneidad } \\
\text { media, grumosidad baja. }\end{array}$ & $\begin{array}{c}\text { Densidad media y homogeneidad } \\
\text { alta, grumosidad baja. }\end{array}$ \\
\hline 21 & A3B1C3 & $\begin{array}{l}\text { Densidad media, homogeneidad } \\
\text { alta, grumosidad baja. }\end{array}$ & $\begin{array}{l}\text { Densidad baja, homogeneidad } \\
\text { alta, grumosidad baja. }\end{array}$ \\
\hline 22 & $\mathrm{~A} 3 \mathrm{~B} 2 \mathrm{C} 1$ & $\begin{array}{l}\text { Densidad alta, homogeneidad } \\
\text { media, grumosidad baja. }\end{array}$ & $\begin{array}{l}\text { Densidad alta, homogeneidad } \\
\text { media, grumosidad alta. }\end{array}$ \\
\hline 23 & $\mathrm{~A} 3 \mathrm{~B} 2 \mathrm{C} 2$ & $\begin{array}{l}\text { Densidad media, homogeneidad } \\
\text { alta, grumosidad baja. }\end{array}$ & $\begin{array}{l}\text { Densidad media, homogeneidad } \\
\text { media, grumosidad baja. }\end{array}$ \\
\hline 24 & $\mathrm{~A} 3 \mathrm{~B} 2 \mathrm{C} 3$ & $\begin{array}{c}\text { Densidad baja, homogeneidad alta, } \\
\text { grumosidad baja. }\end{array}$ & $\begin{array}{l}\text { Densidad baja, homogeneidad } \\
\text { alta, grumosidad baja. }\end{array}$ \\
\hline 25 & A3B3C1 & $\begin{array}{l}\text { Densidad media, homogeneidad } \\
\text { alta, grumosidad baja. }\end{array}$ & $\begin{array}{l}\text { Densidad media, homogeneidad } \\
\text { alta, grumosidad baja. }\end{array}$ \\
\hline 26 & $\mathrm{~A} 3 \mathrm{~B} 3 \mathrm{C} 2$ & $\begin{array}{l}\text { Densidad media, homogeneidad } \\
\text { media, grumosidad baja. }\end{array}$ & $\begin{array}{c}\text { Densidad media, homogeneidad } \\
\text { alta, grumosidad baja. }\end{array}$ \\
\hline
\end{tabular}


ISSN: 2600-5859

\begin{tabular}{|c|c|c|}
\hline 27 & A3B3C3 & $\begin{array}{c}\text { Densidad media, homogeneidad } \\
\text { baja, grumosidad baja. }\end{array}$ \\
\hline
\end{tabular}

A1:2,5 ml de glicerina; A2: 4ml glicerina; A3: 6ml glicerina; B1:0,25 CMC; B2:0,50 CMC; B1:0,75 CMC; $\mathrm{C} 1: 2,5$ ácido acético; $\mathrm{C} 1: 4,0$ ácido acético; $\mathrm{C} 1: 6,0$ ácido acético.

Fuente: Heredia, D.; Pulgar, L. 2019.

Las láminas A3B3C1 y A1B3C2 mostraron las características más adecuadas para su aprovechamiento en la fabricación de polímeros naturales.

\section{Ensayos de degradación de las láminas}

Se evaluó, la degradación en suelo, agua y térmica de las láminas que presentaron mejores características, las tablas siguientes se muestra los resultados:

Tabla 13: Degradación en suelo.

\begin{tabular}{cccccc}
\hline & Método 1 & & & Método 2 & \\
\hline Muestra & $\begin{array}{c}\text { Tiempo } \\
\text { (días) }\end{array}$ & Degradación & Muestra & $\begin{array}{c}\text { Tiempo } \\
\text { (días) }\end{array}$ & Degradación \\
\hline A1 & 140 & Total & D1 & 120 & Total \\
A2 & 140 & Total & D2 & 120 & Total \\
A3 & 140 & Total & D3 & 120 & Total \\
B1 & 140 & Total & E1 & 120 & Total \\
B2 & 140 & Total & E2 & 120 & Total \\
B3 & 140 & Total & E3 & 120 & Total \\
C1 & 140 & Total & F1 & 120 & Total \\
C2 & 140 & Total & F2 & 120 & Total \\
C3 & 140 & Total & F3 & 120 & Total \\
\hline
\end{tabular}

Fuente: Heredia, D.; Pulgar, L. 2019.

En promedio los tiempos de degradación en suelo para las láminas del método 1 y del método 2 fueron de 140 y 120 días respectivamente.

Tabla 14: Degradación en agua.

\begin{tabular}{cccccc}
\hline & Método 1 & \multicolumn{3}{c}{ Método 2 } \\
\hline Muestra & $\begin{array}{c}\text { Tiempo } \\
\text { (días) }\end{array}$ & Degradación & Muestra & $\begin{array}{c}\text { Tiempo } \\
\text { (días) }\end{array}$ & Degradación \\
\hline A1 & 35 & Total & D1 & 30 & Total \\
A2 & 35 & Total & D2 & 30 & Total \\
A3 & 35 & Total & D3 & 30 & Total
\end{tabular}


ISSN: 2600-5859

\begin{tabular}{llllll} 
B1 & 35 & Total & E1 & 30 & Total \\
B2 & 35 & Total & E2 & 30 & Total \\
B3 & 35 & Total & E3 & 30 & Total \\
C1 & 35 & Total & F1 & 30 & Total \\
C2 & 35 & Total & F2 & 30 & Total \\
C3 & 35 & Total & F3 & 30 & Total \\
\hline
\end{tabular}

Fuente: Heredia, D.; Pulgar, L. 2019.

En promedio los tiempos de degradación en agua para las láminas del método 1 y del método 2 fueron de 35 y 30 días respectivamente.

Tabla 15: Degradación térmica laminas método 1

\begin{tabular}{cccc}
\hline Muestra & $\begin{array}{c}\text { Temperatura de } \\
\text { degradación }\left({ }^{\circ} \mathrm{C}\right)\end{array}$ & $\begin{array}{c}\text { Variación de tiempo } \\
\text { (hora) }\end{array}$ & Fracturas \\
\hline $\mathrm{A} 1$ & $60-155$ & 2 & $\mathrm{Si}$ \\
$\mathrm{A} 2$ & $60-155$ & 2 & $\mathrm{Si}$ \\
$\mathrm{A} 3$ & $60-155$ & 2 & $\mathrm{Si}$ \\
$\mathrm{B} 1$ & $60-155$ & 2 & $\mathrm{Si}$ \\
$\mathrm{B} 2$ & $60-155$ & 2 & $\mathrm{Si}$ \\
$\mathrm{B} 3$ & $60-155$ & 2 & $\mathrm{Si}$ \\
$\mathrm{C} 1$ & $60-155$ & 2 & $\mathrm{Si}$ \\
$\mathrm{C} 2$ & $60-155$ & 2 & $\mathrm{Si}$ \\
$\mathrm{C} 3$ & $60-155$ & 2 & $\mathrm{Si}$ \\
\hline
\end{tabular}

Fuente: Heredia, D.; Pulgar, L. 2019.

Tabla 16: Degradación térmica (almidón extraído por el método 2).

\begin{tabular}{cccc}
\hline Muestra & $\begin{array}{c}\text { Temperatura de } \\
\text { degradación }\left({ }^{\circ} \mathrm{C}\right)\end{array}$ & $\begin{array}{c}\text { Variación de tiempo } \\
\text { (hora) }\end{array}$ & Fracturas \\
\hline D1 & $60-120$ & 2 & $\mathrm{Si}$ \\
D2 & $60-120$ & 2 & $\mathrm{Si}$ \\
D3 & $60-120$ & 2 & $\mathrm{Si}$ \\
E1 & $60-120$ & 2 & $\mathrm{Si}$ \\
E2 & $60-120$ & 2 & $\mathrm{Si}$ \\
E3 & $60-120$ & 2 & $\mathrm{Si}$ \\
F1 & $60-120$ & 2 & $\mathrm{Si}$ \\
F2 & $60-120$ & 2 & $\mathrm{Si}$ \\
F3 & $60-120$ & 2 & $\mathrm{Si}$ \\
\hline
\end{tabular}

Fuente: Heredia, D.; Pulgar, L. 2019.

En promedio las temperaturas de degradación para las láminas del método 1 y del método 2 de 150 y $120^{\circ} \mathrm{C}$ respectivamente. 


\section{Propiedades funcionales de las láminas.}

Se evaluó la solubilidad en agua, la absorción de agua y la temperatura de gelatinización, obteniéndose los siguientes resultados:

Tabla 17: Solubilidad en agua, láminas del método 1.

\begin{tabular}{cc}
\hline Muestra & Solubilidad (\%) \\
\hline A1 & 85,6 \\
A2 & 84,2 \\
A3 & 85,2 \\
B1 & 84,7 \\
B2 & 85,1 \\
B3 & 84,4 \\
C1 & 84,7 \\
C2 & 85,1 \\
C3 & 84,7 \\
Promedio & $\mathbf{8 5 , 0}$
\end{tabular}

Fuente: Heredia, D.; Pulgar, L. 2019.

Tabla 18: Solubilidad en agua, láminas del método 2.

\begin{tabular}{cc}
\hline Muestra & Solubilidad $(\%)$ \\
\hline D1 & 77,3 \\
D2 & 76,9 \\
D3 & 76,4 \\
E1 & 76,9 \\
E2 & 76,1 \\
E3 & 76,7 \\
F1 & 76,5 \\
F2 & 76,4 \\
F3 & 76,3 \\
Promedio & $\mathbf{8 5 , 1}$ \\
\hline
\end{tabular}

Fuente: Heredia, D.; Pulgar, L. 2019.

Tabla 19: Absorción de agua, láminas del método 1.

\begin{tabular}{cc}
\hline Muestra & Absorción de agua $(\mathbf{g} / \mathbf{g})$ \\
\hline D1 & 0,6 \\
D2 & 0,6 \\
D3 & 0,6 \\
E1 & 0,6 \\
E2 & 0,6 \\
\hline
\end{tabular}




\begin{tabular}{cc}
\hline E3 & 0,6 \\
F1 & 0,6 \\
F2 & 0,6 \\
F3 & 0,5 \\
Promedio & $\mathbf{0 , 6}$ \\
\hline
\end{tabular}

Fuente: Heredia, D.; Pulgar, L. 2019.

Tabla 20: Absorción de agua, láminas del método 1.

\begin{tabular}{cc}
\hline Muestra & Absorción de agua $(\mathbf{g} / \mathbf{g})$ \\
\hline A1 & 0,8 \\
A2 & 0,8 \\
A3 & 0,8 \\
B1 & 0,8 \\
B2 & 0,7 \\
B3 & 0,8 \\
C1 & 0,7 \\
C2 & 0,7 \\
C3 & 0,6 \\
Promedio & $\mathbf{0 , 7}$ \\
\end{tabular}

Fuente: Heredia, D.; Pulgar, L. 2019.

Tabla 21: Temperatura de gelatinización, láminas dos métodos.

\begin{tabular}{cccc}
\hline \multicolumn{2}{c}{ Método 1 } & \multicolumn{2}{c}{ Método 2 } \\
\hline Muestras & $\begin{array}{c}\text { Temperatura } \\
\left({ }^{\circ} \mathbf{C}\right)\end{array}$ & Muestras & $\begin{array}{c}\text { Temperatura } \\
\left({ }^{\circ} \mathbf{C}\right)\end{array}$ \\
\hline A1 & 155 & D1 & 120 \\
A2 & 154 & D2 & 119 \\
A3 & 154 & D3 & 120 \\
B1 & 154 & E1 & 119 \\
B2 & 155 & E2 & 119 \\
B3 & 154 & E3 & 119 \\
C1 & 155 & F1 & 120 \\
C2 & 155 & F2 & 119 \\
C3 & 154 & F3 & 120 \\
Promedio & $\mathbf{1 5 4 , 4}$ & Promedio & $\mathbf{1 1 9 , 4}$ \\
\hline \multicolumn{3}{c}{} \\
\hline
\end{tabular}

Fuente: Heredia, D.; Pulgar, L. 2019.

\section{Resultados ANAVA, propiedades de las láminas elaboradas.}

A continuación, se resumen de resultados significativos: 
Tabla 22: ANAVA, láminas dos métodos.

\begin{tabular}{|c|c|c|c|c|c|c|}
\hline Método & \multicolumn{2}{|c|}{ Análisis } & $\begin{array}{c}\text { Fisher } \\
\text { calculado }\end{array}$ & p-valor & Medias & Significancia \\
\hline 1 & \multirow{6}{*}{ Propiedades } & \multirow{2}{*}{$\begin{array}{l}\text { Solubilidad } \\
\text { en agua }\end{array}$} & \multirow[b]{2}{*}{258,06} & \multirow[b]{2}{*}{0,0001} & 85,0 & \multirow[b]{2}{*}{$* *$} \\
\hline 2 & & & & & 76,9 & \\
\hline 1 & & \multirow{2}{*}{$\begin{array}{c}\text { Absorción de } \\
\text { agua }\end{array}$} & \multirow[b]{2}{*}{19,16} & \multirow[b]{2}{*}{0,0119} & 0,6 & \multirow[b]{2}{*}{$*$} \\
\hline 2 & & & & & 0,7 & \\
\hline 1 & & \multirow{2}{*}{$\begin{array}{c}\text { Temperatura } \\
\text { de } \\
\text { gelatinización }\end{array}$} & \multirow[b]{2}{*}{6216,57} & \multirow[b]{2}{*}{$<0,0001$} & 154,5 & \multirow[b]{2}{*}{$* *$} \\
\hline 2 & & & & & 119,6 & \\
\hline
\end{tabular}

Fuente: Heredia, D.; Pulgar, L. 2019.

Las láminas con menor contenido de humedad tardan más tiempo en degradarse y dependen del método de extracción, corroborándose lo publicado por (Rosales, 2016), "a mayor porcentaje de humedad menor tiempo de degradación”.

De acuerdo al análisis estadístico la solubilidad en agua de las láminas depende del método de extracción, en el caso de las láminas elaboradas para los ensayos se adicionó un plastificante que de acuerdo a (Oropeza, Montes y Padrón, 2016, p. 65-93) "la solubilidad se relaciona con el plastificante".

La absorción de agua de las láminas también depende del método de extracción, aquellas del método 1 con mayor contenido de plastificante absorben menor cantidad de agua a diferencia de las láminas del método 2 con menor cantidad de plastificante, según (Oropeza, Montes y Padrón, 2016, p. 65-93), "altas concentración del plastificante disminuyen la absorción de agua”.

La gelatinización de las láminas del método 1 necesitan mayor temperatura para que susciten y sus propiedades elásticas se debiliten, según literatura reportada (González, 2018, p. 3544), la temperatura actúa como deshidratante y los polímeros naturales se vuelen cada vez más duros y quebradizos..

\section{Conclusiones}

- El mayor rendimiento en extracción de almidón se obtuvo con el método 1 que utiliza ácido cítrico al 3\%, Utilizando el método de extracción de almidón de mayor rendimiento cercano al $28 \%$, adecuado para su aplicación a mayor escala. 
- El almidón obtenido de la malanga blanca puede formar láminas poliméricas con características adecuadas para producir polímeros naturales al mezclarlo con diferentes dosificaciones de aditivos y todas tienen efectiva degradabilidad.

- Las características del almidón obtenido y de las láminas elaboradas a partir del mismo están directamente relacionadas con el método de extracción utilizado, pudiéndose adecuar a las necesidades del productor.

- La simplicidad y economía de los procesos de extracción utilizados viabilizan su aplicación en las comunidades productoras de malanga.

- La producción de almidón de malanga blanca es ambientalmente favorable por sus potenciales aplicaciones en alternativa al uso de polímeros sintéticos y socialmente factible ya que existe un alto porcentaje de rechazo de malanga en el proceso de selección de producto para la exportación.

\section{Referencias bibliográficas.}

Álvarez, R., Rondón, C., Gutiérrez, F., Aguilar, C., Suárez, I. y Freddy, H. (2017). "Alternativa ecológica en la obtención de un polímero biodegradable a partir del almidón de yuca dulce". Agrollanía,. vol. 14, no. enero-diciembre, pp. 37-44.

Ambiente, C.D.E.M. (2017)."En medio ambiente tema: Elaboración de láminas biodegradables a partir de los residuos del almidón de yuca ( Manihot esculenta ) Rodríguez Mendoza Elvin Daniel tutora :". pp. 50.

Angeles, C.(2015). "Diseño de un proceso industrial para obtener plástico biodegradable (tps) a partir de almidón de yuca manihot sculenta". No. February, pp. 9-11.

Anne, H. (2012)."The mystery of malanga: possible roles of xanthosoma violaceum in ancient maya diet, culture, and agriculture". [en línea]. disponible en: https://scholar.colorado.edu/anth_gradetds/29.

Avil, R. (2005). "Polímeros biodegradables ".Vol. 1, pp. 15-22.

Beltrán, M. (2012). "Estructura y propiedades de los polímeros". pp. 1-42.

Bianco, H., Capote, T. y Garbendia, C. (2014). "Determinación de humedad en harina precocida de maíz blanco utilizando un horno de microondas doméstico". Revista del Instituto Nacional de Higiene «Rafael Rangel», Vol. 45, No. 2, pp. 29-36. 
Biodegradáveis, F.(2012)."Composición y procesamiento de starch-based biodegradable films . composição e transformação". , Vol. 10, No. 1, pp. 182-192.

Brown, P., Forrest, M. (2000)."Rapra review reports 10". [en línea], vol. 14, no. 3. disponible en:

http://scholar.google.com/scholar?hl=en\&btng=search\&q=intitle:rapra+review+re+ports\#2.

Carballo, B. (2017). "Obtención y caracterización de almidones termoplásticos obtenidos a partir de almidones injertados con poliésteres biodegradables". [en línea]. pp. 91. disponible en:

https://cicy.repositorioinstitucional.mx/jspui/bitstream/1003/466/1/pcm_d_tesis_2017_cuev as_zujey.pdf.

Chávez, S. (2015). "Obtención de almidón modificado a partir de tres cultivares de musáceas". pp. 39.

Domínguez, M.; Jimenez, M. (2012). "Películas comestibles formuladas con polisacáridos : propiedades y aplicaciones". temas selectos de ingeniería de alimentos [en línea]. Vol. 6, No. 2, pp. 110-121. disponible en: mariaf.dominguezcy@udlap.mx.

Ebnesajjad, S. (2013). Handbook of biopolymers and biodegradable plastics [en línea]. s.l.: s.n. 2013. isbn 9781455728343 . disponible en:

http://www.sciencedirect.com/science/article/pii/b9781455728343000094.

González, J. (2018) "Análisis comparativo de los métodos húmedo y alcalino en la extracción de almidón de semillas de Amaranthus quitensis L.". Revista del instituto de investigación de la facultad de ingeniería geológica, minera, metalurgica y geográfica. Vol. 21, No. 41, pp. 35-44.

Gorgonio, I. y Tolentino, L. (2012)."Especialización en química aplicada". [en línea], 2012. disponible en:

https://ciqa.repositorioinstitucional.mx/jspui/bitstream/1025/371/1/gorgonio_ lopez tolentino.pdf.

Guevara, S. (2016)."Elaboración y evaluación nutricional de cupcake funcional a base de harina de arveja (pisum sativum) y harina de trigo (triticum aestivum), para fortalecer la dieta diaria.". facultad de ciencias [en línea]. Vol. bachelor, pp. 73. disponible en: http://dspace.espoch.edu.ec/handle/123456789/4947.

Haro-Velasteguí ', A.J., Borja-Arévalo ", A.E. y Triviño-Bloisse "I, S.Y. (2017) "Análisis sobre el aprovechamiento de los residuos del plátano, como materia prima para la 
producción de materiales plásticos biodegradables analysis on the use of banana waste, as raw material for the production of biodegradable plastic materials análise". Vol. 3, No. 2, pp. 506-525.

Heredia, D., Pulgar, L.(2019). "Comparación de dos métodos de extracción de almidón de colocasia esculenta (malanga blanca) para la obtención de un polímero biodegradable" disponible en: http://dspace.espoch.edu.ec/handle/123456789/13256

INEN. (2013). "Norma técnica ecuatoriana NTE INEN 520: Primera revisión harinas de origen vegetal. determinación de la ceniza".

INEN. (1981). "Norma técnica ecuatoriana NTE INEN 0522 harinas de origen vegetal. determinación de la fibra cruda". , 1981. Vol. 0522.

Marcos, C. (2018). Extracción de almidón a partir de residuos de banano (musa paradisiaca) para la elaboración de un biopolímero. s.l.: s.n. isbn 1400674840.

Meneses, J., Corrales, C. y Valencia, M. (2007). "Síntesis y caracterización de un polímero biodegradable a partir del almidón de yuca". Revista EIA. No. 8, pp. 57-67.

Morillas, A., Valdemar, R., Villavicencio, M. y Pérez, M. (2015). "Bioplásticos y plásticos degradables". Universidad Autonoma Metropolitana. pp. 11.

Niño, K., Huerta, A., Guadalupe, M., Verde, R. y Rodríguez, 1. (2010). "Películas biodegradables a partir de residuos de cítricos: propuesta de empaques activos". arévalo et al., 2010. rev latinoam biotecnol amb algal 1(2):124-134 [en línea]. Vol. 1, pp. 124-134. disponible en:

http://www3.inecol.edu.mx/solabiaa/archivos/documentos/relbaa/arevalo_et_al_revlatinoa mbiotecnolambalgal_v1n2.pdf.

NTE INEN 0519. (1981). "Harinas de origen vegetal. determinación de la proteína". [en línea], 1981. vol. $0519 . \quad$ disponible en: https://ia801902.us.archive.org/25/items/ec.nte.0519.1981/ec.nte.0519.1981.pdf.

NTE INEN 1513. (1987). "Granos y cereales. maíz. determinación del contenido de humedad". [en línea]. Vol. 1513. disponible en: https://ia801902.us.archive.org/26/items/ec.nte.1513.1987/ec.nte.1513.1987.pdf.

Oropeza, R., Montes, A. y Padrón, C. (2016). "Películas biodegradables a base de almidón: propiedades mecánicas, funcionales y biodegradación biodegradable". revista venezolana de ciencia y tecnología de alimentos [en línea]. Vol. 7, No. 1, pp. 65-93. disponible en: http://www.unicolmayor.edu.co/invest_nova/nova/nova14_artorig4.pdf. 
Pillajo, R. (2016). "Aplicación y utilización gastronómica de la malanga". [en línea]. disponible en: http://200.24.220.94/handle/33000/5960.

Râpă, M., Grosu, E., Andreica, M. y Hetvary, M. (2014). "Polyvinyl alcohol and starch blends : properties and biodegradation behavior". ecoterra -journal of environmental research and protection. Vol. 11, No. 1, pp. 34-42.

Rapelo, A., Castillo, P. y Lengua, M. (2013). "Propiedades fisicoquímicas, morfológicas y funcionales del almidón de malanga (colocasia esculenta)". revista lasallista de investigación. Vol. 10, No. 2, pp. 52-61.

Rosales, A. (2016). "Departamento de química título: obtención de biopolímero plástico a partir del almidón de malanga ( colocasia esculenta ), por el método de polimerización por condensación en el mayo - abril 2016".

Salvador, U. (2013). "Extracción de almidón a partir de guineo majoncho verde ( musa sp . variedad cuadrado )".

Sánchez, A., Contreras-Esquivel, J., Nevárez-Moorillón, G. y Aguilar, C. (2015). "Caracterización de películas comestibles a base de extractos pécticos y aceite esencial de limón mexicano". cyta - journal of food [en línea]. Vol. 13, No. 1, pp. 1725. disponible en: http://dx.doi.org/10.1080/19476337.2014.904929.

Sudhakar, Y., Selvakumar, M. y Bhat, D. (2018). "Methods of preparation of biopolymer electrolytes". biopolymer electrolytes. pp. 35-52.

Valero-Valdivieso, M., Ortegón, Y. y Uscategui, Y. (2013). "Biopolímeros: avances y perspectivas". dyna (colombia). Vol. 80, No. 181, pp. 171-180.

Velasco, R., Enriquez, M., Torres, A., Palacios, L. y Ruales, J. (2012). "Caracterización morfológica de películas biodegradables a partir de almidón modificado de yuca, agente antimicrobiano y plastificante". biotecnología en el sector agropecuario y agroindustrial: bsaa. Vol. 10, No. 2, pp. 152-159.

Villada, H., Acosta, H. y Velasco, R. (2008). "Investigación de almidones termoplásticos, precursores de productos biodégradables". Información tecnológica. Vol. 19, No. 2, pp. 3-14.

Zapata, J. y Velásquez, C. (2013). "Estudio de la producción y comercialización de la malanga: estrategias de incentivos para la producción en el país y consumo en la ciudad de guayaquil". Universidad Politécnica Salesiana. pp. 172.

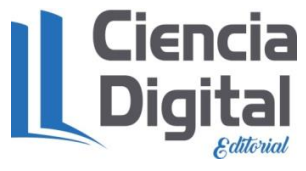


Para citar el artículo indexado.

González García , J. C., Godoy Ponce, S. C., Heredia Jara, A., \& Pulgar Astudillo, L. J. (2020). Extracción de almidón de malanga blanca, una alternativa socioambiental para la producción de bases poliméricas biodegradables. ConcienciaDigital, 3(3),

6-16. https://doi.org/10.33262/concienciadigital.v3i3.1263

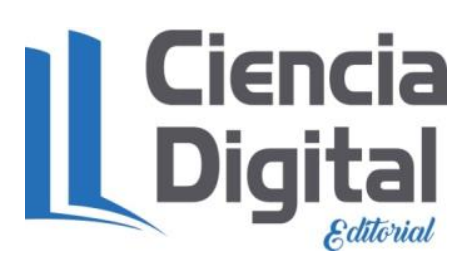

El artículo que se publica es de exclusiva responsabilidad de los autores y no necesariamente reflejan el pensamiento de la Revista Conciencia Digital.

El articulo queda en propiedad de la revista y, por tanto, su publicación parcial y/o total en otro medio tiene que ser autorizado por el director de la Revista Conciencia Digital.
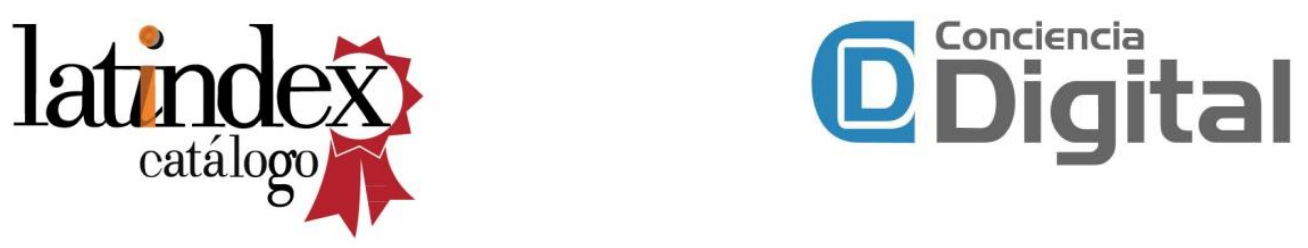